\title{
ESTRATEGIAS REGIONALES DE GESTIÓN AMBIENTAL. EL CASO DEL PROYECTO MINERO LAS BAMBAS DE APURÍMAC
}

\author{
Nemesio Espinoza Herrera* \\ E-mail: nespinozah@hotmail.com \\ Mauro Mariano Astocondor*** \\ E-mail: mmarianoa@unmsm.edu.pe \\ José Porlles Loarte***** \\ E-mail: joseporlles@yahoo.com \\ Alfonso Romero Baylón ${ }^{* * * * * *}$ \\ E-mail:aromerob@unmsm.edu.pe
}

\begin{abstract}
RESUMEN
Objetivo: El estudio tiene por objetivo evaluar las incidencias de la actividad minera en la ecología regional y proponer una estrategia de gestión ambiental. Método: Se ha visitado la mina Las Bambas ubicada en la región de Apurímac así como a las comunidades aledañas a ella tales como Tambobamba, Challhuahuacho, Progreso y Chuquibambilla, lugares que están ubicados en las provincias de Cotabambas y Grau respectivamente. Se ha entrevistado a los alcaldes provinciales de Cotabambas y de Grau. Así mismo se han hecho entrevistas en profundidad a los comuneros. A través de las visitas efectuadas se han hecho observaciones directas de la realidad. Resultado: En la región Apurímac existen evidencias de contaminación ambiental y se prevé que la explotación minera del proyecto Las Bambas agravará aún más el problema. Al no existir una estrategia regional de gestión ambiental se pondrá en grave riesgo la biodiversidad existente. Conclusión: Siendo necesaria la explotación minera para promover el desarrollo económico y social de esta región calificada de extrema pobreza, se requiere el establecimiento de una estrategia regional de gestión ambiental.
\end{abstract}

Palabras Clave: Gestión ambiental, minería.

\section{ABSTRACT}

Objetive: The study has as purpose the evaluation of incidences in mining activity in regional ecology and to propose an enviromental management strategy. Method: We have been visited the Las Bambas mine located in Apurimac as well as the surrouding communities such as Tambopata, Challhuahuacho, Progreso y Chuquibambilla, places that are located in the provinces of Cotabambas and Grau respectively. It has been interviewed the provincial majors of Cotabambas and Grau. Similarily, deeply interviews to

* Magíster en Administración. Licenciado en Administración. Responsable de la Investigación. Profesor Principal de la Facultad de Ciencias Administrativas (UNMSM).

* Magíster en recursos acuáticos. Biólogo. Profesor Asociado de la Facultad de Ciencias Administrativas (UNMSM).

萧 Magíster en Administración. Ingeniero Químico. Profesor Asociado de la Facultad de Ciencias Administrativas (UNMSM).

Ingeniero de Minas. Profesor Auxiliar de la Facultad de Ciencias Administrativas (UNMSM). 
the community people have been done. Through the visits it has been made observations related to the reality. Results: In the Apurimac region there are evidences of enviromental contamination and is foreseeable that the mining operations of the project Las Bambas will worsen the problem. As there is not a regional strategy of enviromental management it will cause a great risk to the existing biodiversity. Conclusion: Being necessary the mining processes in order to promote the social and economic development of the region labeled as extreme poverty, the region of Apurimac requires the establish of a regional strategy for environmental management.

Key Words: Environmental management, mining industry.

\section{INTRODUCCIÓN}

El mega proyecto minero Las Bambas, a cargo de la empresa suiza Xstrata Copper, se encuentra ubicado entre las provincias de Cotabambas y Grau de la región Apurímac. Esta región ha sido declarada como «Reserva Minera Nacional» por existir indicios de una gran potencialidad minera. El proyecto concedido se encuentra a una altitud aproximada de 4,500 msnm y abarca 35,000 Has. La región Apurímac, especialmente las provincias donde se encuentra ubicada la mina, son zonas calificadas como de extrema pobreza.

La concesión de este gran yacimiento minero y su explotación en un futuro cercano (pues actualmente la mina está en la fase de exploración y factibilidad), traerá consigo la posibilidad de promover el desarrollo de la región de manera sostenida y sostenible. Por lo pronto, la suscripción del contrato entre el gobierno peruano y la empresa minera ganadora de la licitación internacional, producida en octubre del 2004, ha significado la entrega de 121 millones de dólares, de los cuales 91 millones han sido ya entregados a través de un fedeicomiso y la mitad de ellos se han destinado para financiar proyectos sostenidos de desarrollo social en la zona, que actualmente se encuentran en ejecución en las diferentes localidades de las provincias de Grau y Cotabambas ${ }^{1}$.

Sin embargo, si bien el proyecto minero Las Bambas constituye una buena oportunidad para promover el desarrollo de los pueblos, también es cierto que, de no existir una estrategia bien concebida y concertada, inevitablemente va a ocasionar graves efectos en el medio ambiente. Precisamente, esta investigación tiene por propósito efectuar una evaluación medioambiental, predecir los efectos de la explotación minera contra el ecosistema y establecer lineamientos de una estrategia regional de gestión ambiental.

\section{PLANTEAMIENTO DEL PROBLEMA}

Algunos datos ilustran las condiciones de extrema pobreza de la región Apurímac, que tiene una población total de 426,904 habitantes.

- Esperanza de vida: 64 años en promedio.

- Servicios: el 54\% de la población se encuentra sin agua potable y el $80 \%$ no tiene servicios de saneamiento. El 55\% de la población no tiene alumbrado eléctrico.

- Educación: el 30\% de la población es analfabeta.

- Salud: el $45 \%$ de los niños menores de 5 años tienen desnutrición severa, el $87 \%$ de la población enferma hizo consultas médicas el año pasado; existen tres médicos por cada 10 mil habitantes.

La provincia de Cotabambas tiene 44,069 habitantes con 119 nuevos soles de ingreso familiar per cápita, mientras que la provincia Grau tiene una población de 27508 habitantes con 130 soles al mes de ingreso familiar per cápita²

Según declaraciones del Alcalde Provincial de Cotabambas, Luis Calderón Jara, tienen 54 mil habitantes, de los cuales el $61 \%$ es analfabeto. Sólo tienen cinco médicos en toda la provincia y la desnutrición alcanza el 52\% de la población. En cuanto a servicios básicos, solo el $30 \%$ puede acceder a ellos.

Por otra parte, en lo que respecta a las potencialidades mineras de las dos provincias de Apurímac en las que se encuentra la mina Las Bambas (Cotabambas y Grau), se debe manifestar que de ellas se tiene noticias desde 1911. Se trata de una gran y vasta zona minera que comprende los yacimientos de Chalcobamba, Ferrobamba, Sulfobamba, Charcas y Azuljaja. Las Bambas puede alcanzar una inversión cercana a los dos mil millones de dólares y crear miles de puestos de 
trabajo. Es evidente que estos testimonios acreditan que la región Apurímac es, en efecto, una gran reserva minera nacional que requiere necesariamente de capital extranjero y tecnología de punta para que el Perú se consolide como país minero.

El gran proyecto minero Las Bambas comprende yacimientos de cobre con presencia de oro, molibdeno, plata y hierro. Los yacimientos de cobre son del tipo skarn (rocas con minerales como calcio, magnesio y hierro). Las reservas probadas se calculan en 40.5 millones de toneladas de mineral, con leyes mayores a $2 \%$ de cobre, y un potencial de exploración superior a los $500 \mathrm{mi}$ llones de toneladas. Si bien los yacimientos son básicamente de cobre (con contenidos promedio de $4.85 \%$ de ese mineral), diversos estudios revelaron también la presencia de 0.43 gramos de oro por tonelada y 26.65 gramos de plata por tonelada. Más de siete mil 500 metros de perforaciones diamantinas se realizaron como parte del programa de exploraciones en Las Bambas, desde 1966. Estudios geológicos refieren que se situaría en la continuación del Copper Belt del Sur, zona en la que se hallan los importantes yacimientos cupríferos de La Escondida y Chuquimata en Chile; Cuajone, Toquepala, Tintaya, Quechuas, Antapacca y Los Chancas, en el Perú ${ }^{3}$.

De otro lado es necesario destacar que la explotación de las riquezas naturales en general trae consigo, inexorablemente, la degradación del medio ambiente. En lo que respecta a la explotación minera, la contaminación generada reviste aún mayor gravedad por las evidentes secuelas negativas de la contaminación ambiental de orden físico (radiación solar, aire, suelo, agua, etc.) y biológico (plantas, animales, personas). Por tales razones, la humanidad empieza a manifestar sus preocupaciones en torno a la degradación atmosférica y de la biodiversidad configurándose toda una megatendencia universal por la conservación medioambiental y por la ecología, que posibilite establecer un equilibrio armónico entre el hombre y su medio en la búsqueda del progreso de la humanidad.

La gestión ecológica -proceso destinado a resolver el gran problema mundial de la contaminación ambiental- constituye uno de los elementos más importantes de la actual cultura de las organizaciones, que es imposible ignorar por cuanto la asunción plena de sus responsabilidades sociales de no contaminar el medio ambiente a través de la concepción e implementación de estrategias y sistemas de gestión medioambiental así como con la ejecución de periódicas auditorías ecológicas, les permite generar ventajas competitivas para la conquista de mercados mundiales y para el logro de mejores condiciones en la convivencia humana. La actividad minera en la región Apurímac afectará inevitablemente al medio ambiente físico y biológico cuando menos en la formación de sustancias ácidas con contenidos metálicos tóxicos tales como el cadmio, mercurio, arsénico, selenio, zinc, plomo, etc.; generación de aceites y disolventes contaminantes; emisión de gases, polvos, vibraciones, humos y ruidos; generación de oxidaciones de minerales que no se pueden disolver, lo que ocasiona la presencia de desechos orgánicos e inorgánicos (basura); contaminación de los recursos hídricos (ríos, lagos, mares), sobreexplotación del agua y la generación de aguas residuales. Asimismo se destruirían paisajes naturales, se alteraría la identidad cultural de grupos humanos, además de afectar al patrimonio cultural. En suma, la minería destruye la atmósfera y la biodiversidad.

Es en el contexto de estos tres problemas vinculantes señalados (extrema pobreza de la región Apurímac, potencialidad minera de la región y degradación ambiental) que se plantea la necesidad de la explotación minera siempre y cuando se tomen actitudes vigilantes para que la empresa minera haga uso de las tecnologías modernas para el control del impacto ambiental, respetando la identidad de los pueblos y, sobre todo, adoptando actitudes positivas y razonadas para exigir la puesta en marcha de un sistema de gestión ecológica regional concertado, destinado a resolver el gran problema de la contaminación ambiental en Apurímac.

\section{MÉTODOS}

Para fines de la investigación se ha visitado la mina y las zonas aledañas (Tambobamba, Challhuahuacho, Progreso y Chuquibambilla), en dos ocasiones (agosto y noviembre), lugares que están ubicados en las provincias de Cotabambas y Grau respectivamente $^{4}$. En ambas visitas se han efectuado observaciones directas; entrevistas en profundidad a comuneros, autoridades locales, comunales y educativas, y lecturas de documentos relacionados al tema de la investigación. Se ha entrevistado al Alcalde Provincial (e) de 
Cotabambas (Prof. Gumercindo Huanca Villancal) y al Alcalde Provincial de Grau (Prof. Milder Tuiro Hurtado).

\section{RESULTADOS, ANÁLISIS Y DISCUSIÓN}

1. Las zonas en las que se encuentra la mina Las Bambas forman parte de las praderas altoandinas del Perú y constituyen cabeceras de cuencas que posibilitan el ciclo hídrico. En general, son ecosistemas frágiles. Se ubican entre los 4,200 y 4,800 msnm. Las precipitaciones se producen en verano (enero a marzo) y se manifiestan como lluvias de la sierra que discurren como aguas pluviales de escorrentía. De mayo a octubre la zona se presenta seca. En la zona existen acuíferos que aseguran la dotación de agua de manera permanente (tanto superficiales como subterráneas), representados en lagunas y bofedales. Todas estas características naturales de la zona minera de Las Bambas, sumadas a la vulnerabilidad del ecosistema, ponen de manifiesto la importancia de desarrollar políticas y estrategias de gestión ambiental. Según el informe de D \& E Desarrollo y Ecología SAC., empresa consultora encargada de hacer el estudio de impacto ambiental para la empresa minera,

«El área del proyecto presenta pendientes abruptas tanto al este como al oeste del cerro Pichacani; éstas pendientes se vuelven mas fuertes y pronunciadas desde la Quebrada Huascachaca hasta el poblado de Challhuahuacho, y se presentan paralelas al río Fuerabamba, encajonándolo y formando así un valle en la parte baja del proyecto».

2. La mineralización de cobre de la zona está principalmente emplazada en los contactos de calizas y rocas intrusivas de la Era Terciaria. Las calizas corresponden a la Era Cretácea, similar a los depósitos de Skarn en Tintaya y Antamina. La forma de mineralización más común es la calcopirita que contiene oro y plata asociados con cobre. Aunque varios minerales metálicos han sido encontrados en Las Bambas, solamente algunos minerales ocurren en cantidades económicamente rentables y casi exclusivamente en el cuerpo de Skarn. Todo esto resulta claro indicio de que también en otras zonas de la región de Apurímac (declarada Reserva Minera Nacional) existen grandes reservas mineras.
3. La ejecución del proyecto minero Las Bambas es una buena oportunidad para promover el desarrollo económico y social sostenido (y sostenible) de la región y, por ende, del país. Si bien la mayoría de la población está de acuerdo con la explotación minera, existen otros sectores que se oponen activamente a la explotación minera, con argumentos discordantes con la modernidad.

4. En la región Apurímac, y en especial en las provincias en las que se encuentra la mina Las Bambas, no existe conciencia sobre la importancia de la conservación del medio ambiente tanto en la población en general como en las autoridades regionales, locales y comunales. Todo esto demuestra que existe la necesidad de emprender estrategias de concientización y sensibilización en cuanto a la conservación del medio ambiente como un tema de sobrevivencia de la humanidad.

5. No obstante a que la región Apurímac es la menos contaminada en comparación con otras, sin embargo en esta región ya existen aún antes de la explotación minera- indicios de contaminación ambiental originados principalmente por la afluencia de desaguies de las ciudades y poblados hacia los ríos; por la carencia de sistemas de manejo de la basura; así como por las paupérrimas condiciones de salubridad (falta de agua y desaguie, falta de servicios higiénicos, inexistencia de una educación y cultura proactiva de salud, etc.).

6. En la región Apurímac, tal como fue constatado a través de las breves visitas hechas también a otras provincias de la región tales como Abancay y Antabamba por tratarse de un estudio de ámbito regional, se aprecia que tanto la población en general como las autoridades regionales, locales y comunales, desconocen la importancia de conservar el medio ambiente. Ello se corrobora con la inexistencia de información vinculada a la conservación medioambiental en la página Web del Gobierno Regional de Apurímac y de las provincias de la región.

7. La necesaria explotación de la potencialidad minera de Apurímac implica, sin embargo, que la población regional y nacional tomen actitudes vigilantes para que las empresas mineras hagan uso de las tecnologías modernas para el control del impacto ambiental y respeten la identidad de los pueblos. 
8. Teniendo en cuenta que la actividad minera aún no se realiza en la mina Las Bambas por estar en la fase de exploración, se prevé que la explotación minera generará inevitablemente mayor contaminación ambiental zonal y regional que, de no existir una estrategia de gestión ambiental, pondrá en grave riesgo la biodiversidad existente en la región. Es necesario atenuar estos impactos mediante estrategias integrales de gestión ecológica que posibiliten la explotación de los recursos naturales con el menor daño posible al ecosistema y dentro del marco de respeto a la vulnerabilidad del medio ambiente. Así mismo existen antecedentes de serios problemas ocurridos en las zonas aledañas a las minas Antamina (en Ancash), y Yanacocha (en Cajamarca), donde el daño al ecosistema ocasionó enfrentamientos con las comunidades locales.

9. En otras palabras, frente a la necesidad de la explotación no sólo de las minas sino en general de los recursos naturales (turísticos y agropecuarios) de Apurímac que significan serios impactos ambientales, se hace necesario establecer estrategias regionales de gestión ambiental en el marco del proceso de descentralización nacional. Para ello será necesario la participación activa de las comunidades organizadas, instituciones universitarias, investigadores, autoridades (comunales, locales, regionales y del Estado), profesionales y ciudadanos en general para promover una cultura sostenida de explotación de sus recursos pero dentro del marco de respecto al medio ambiente regional ${ }^{5}$.

10. La concepción de una estrategia regional de gestión ambiental debe tener en consideración un conjunto de factores interdisciplinarios y por lo tanto debe contar con la participación de profesionales de diversas especialidades (hidrología, hidrogeología, biología, química, gestión, ingeniería civil, estadística, economía, sociología).

\section{CONCLUSIONES}

1. La explotación de la mina Las Bambas no sólo es viable sino una necesidad histórica para promover el desarrollo económico y social de la región de Apurímac, actualmente calificada como de extrema pobreza.
2. La explotación minera en Las Bambas, que se ha de producir dentro de uno o dos años, generará las siguientes posibles consecuencias en la degradación del medio ambiente: residuos sólidos, erosión, depósito de sedimentos, deslizamientos, colapsos del suelo, movimiento de aguas subterráneas, cambios de temperatura, drenajes (desaguies, derrames, vaciados), deterioro de paisajes, lesiones pulmonares en personas y animales, sedimentos hacia los ríos, cambios de velocidad de las corrientes de agua, migración de especies animales, degradación de valores y costumbres (bares, prostitución, etc.) que afectarán los recursos etnológicos, las tradiciones, fiestas religiosas y costumbristas, etcétera.

3. La región Apurímac requiere una estrategia regional de gestión ambiental concertada, en la que no solamente se consideren los efectos de la actividad minera sino en general los efectos negativos al medioambiente, en el marco de un proceso sostenido de desarrollo económico y social de la región.

4. Es necesario realizar la difusión a nivel regional del Estudio de Impacto Ambiental (EIA) que la empresa D \& E Desarrollo y Ecología SAC. efectuara por encargo de la empresa Xstrata Perú S.A., así como difundir la ejecución de diversos estudios macroambientales, a fin de sensibilizar a las comunidades y a la población en general sobre la necesidad de la conservación del medio ambiente. Actualmente el Estudio de Impacto Ambiental es absolutamente desconocido por la población y tampoco existen otros estudios al respecto. Todo esto permitirá una mejor respuesta (inteligente y organizada) de las comunidades ante eventuales irresponsabilidades sociales en que incurra la empresa minera, además de fiscalizar el cumplimiento del EIA, gestar una cultura conservacionista, etc. y así evitar experiencias negativas como las ocurridas en otras zonas del país.

5. Se requiere una mayor valoración de los bienes y servicios ambientales de la región (mapeo de las condiciones, componentes y realidades medioambientales), a fin de contribuir a la formulación de políticas y estrategias de gestión regional del medio ambiente.

6. La concepción del sistema, políticas y estrategias de gestión medioambiental de Apurímac 
deben abordar necesariamente el criterio de que el crecimiento de la economía regional debe concordar con el equilibrio ecológico. Esto quiere decir que un nuevo sistema económico sostenido de la región debe estar sustentado en el desarrollo sostenible que implica una nueva concepción de la gestión ambiental, siempre pensando en el bienestar de las futuras generaciones.

7. Toda estrategia regional de gestión ambiental debe tener en cuenta que es necesario establecer mecanismos concertados de reducción de la contaminación en general, no sólo de la actividad minera. De la misma manera debe plantearse la necesidad de la aplicación (y exigencia a la empresa minera como mínima expresión de su responsabilidad social) de tecnología de punta (tecnologías limpias) para mitigar los efectos de la contaminación.

\section{AGRADECIMIENTOS}

Especial agradecimiento al Sr. Gumercindo Huanca, Alcalde interino de la Provincia de Cotabambas y Primer Regidor; al Sr. Milder Tuiro, Alcalde Provincial de Grau; al Sr. Evaristo Mendoza Wilca, comunero de la Comunidad San Juan de Cotabambas, a los comuneros y autoridades comunales de las localidades de Chuquibambilla, Tambobamba, Antabamba y Chalhuahuacho, y a todas las personas con quienes se compartieron nuestras preocupaciones en torno a la contaminación ambiental de la región y de las graves consecuencias a la biodiversidad si es que no se establece una estrategia regional de gestión ambiental.

\section{NOTAS}

1 Actualmente se viene implementando un conjunto de proyectos relacionados al desarrollo social con fondos del fideicomiso. En entrevistas efectuadas con los alcaldes y comuneros (y tal como se ha podido apreciar directamente) se establece que hay servicio de Internet de ancha base, se electrifica a diferentes comunidades, se construyen centros educativos e infraestructuras de saneamiento, puestos de salud, carreteras, etcétera.

2 Fuente: INEI. INEI / PNUD. Sinopsis del desarrollo humano. (2000). Citado por el Prof. Porlles, 2005.

3 Información publicada en la página web de PROINVERSIÓN. Ver www.pronversión.gob.pe.

4 La mejor ruta para llegar de Lima a la mina Las Bambas de Apurímac, es la vía Cuzco-Tambobamba, que es una carretera sin asfaltar, viaje que tiene una duración de aproximadamente ocho horas. De Tambobamba se debe viajar a la comunidad de Challhuahuacho en combi o camión, con salidas irregulares. De esta comunidad al campamento de la mina se pueden utilizar vehículos particulares (taxi). La ruta más difícil y larga es la de Abancay-Grau, con carretera sin asfaltar, por la que llegamos a la ciudad de Chuquibambilla (capital de Grau) en 6 horas aproximadamente. De esta ciudad se debe viajar al distrito de Progreso en vehículos con salidas irregulares y de allí en camiones o camionetas hasta la mina.

5 Téngase en cuenta que existe la denominada «Declaración de Challhuahuacho» (2004), en la que comunidades y funcionarios del gobierno (del Ministerio de Energía y Minas, PROINVERSIÓN) así como autoridades de la Región Apurímac y todos los alcaldes de los distritos involucrados en el proyecto, acuerdan firmar un acta para hacer respetar sus derechos, aprobando la viabilidad de la mina Las Bambas.

\section{BIBLIOGRAFÍA}

Agencia de Promoción de la Inversión Privada para el desarrollo de inversiones en el Perú (PROINVERSION). Ver página web: http:// www.proinversion.gob.pel

Aste Daffós, Juan y Glave, Manuel. (2004). Procesos de concertación y desarrollo local en zonas mineras. Artículo electrónico. Lima.

Consejo Nacional de Ciencia y Tecnología (CONCYTEC). (2005). Plan nacional de ciencia y tecnología e innovación para la competitividad y el desarrollo humano 20062021. Lima, noviembre del 2005. Ver página web: www.concytec.gob.pe.

Consejo Nacional del Ambiente (CONAM). Ver página web: http://www.conam.gob.pe/.

Cortázar, Pedro Felipe. (1969). Documental del Perú-Apurímac. Tercera edición. Lima, Ioppe Editores e Iberia SA.

Espinoza Herrera, Nemesio. (2000). Apurímac, Pobrezas y Riquezas. Lima, Editorial San Marcos EIRL.

Espinoza Herrera, Nemesio. (2003). El desarrollo de Apurímac en el Siglo XXI. Lima. Artículo publicado en la revista Bodas de Oro del Club Departamental de Apurímac.

Gallopin, Gilberto. (2003). Sostenibilidad y desarrollo sostenible, un enfoque de sistemas. En Revista Serie Medio Ambiente y Desarrollo. Chile, 2003.

Hosting, Rainer (compilador) (2002). Bibliografía del Departamento de Apurímac. Lima, CONCYTEC, mayo 2002.

Instituto de Investigación. (2002). Estudio de la Contaminación Ambiental de las minas 
artesanales de oro. En Revista del Instituto de Investigación de la Facultad de Geología Minas, Metalurgia y Ciencias Geográficas de la UNMSM. Volumen $5, \mathrm{~N}^{\circ} 09$, junio 2002.

Instituto Nacional de Estadística e Informática (INEI) (2003). Sinopsis del desarrollo humano 2000. Lima, INEI / PNUD.

Instituto Nacional de Estadística e Informática (INEI). Ver página web: http://www.inei.gob.pe

International Standards Organization (ISO). Ver página web: $w w w . i s o . o r g$

Kuramoto, Juana. (2004). Impacto de la minería: desarrollo insuficiente y deterioro ambiental. En Revista perú económico del grupo de análisis para el desarrollo. Agosto del 2004.

Ministerio de Agricultura (MINAG) e Instituto Nacional de Estadística e Informática (INEI). (20002002). Compendio Estadístico- Apurímac.
Ministerio de Energía y Minas. Ver página web: http://www.minem.gob.pe.

Organización de las Naciones Unidas (ONU). (1992). Cumbre para la Tierra-Conferencia sobre el Medio Ambiente y el Desarrollo. Río de Janeiro, Brasil. Junio de 1992.

Padilla, Eusebio Dionisio y López Mas, Julio. (2004). Sostenibilidad ambiental y responsabilidad social en la actividad aurífera: el caso de Pierina (Ancash). Proyecto de Investigación e Informe de Avance presentado al Taller de Investigación 2004 de la Facultad de Ciencias Administrativas (UNMSM).

Página web: http://www.gestion-ambiental.com.es.

Proyecto Las Bambas. Ver página web: www.lasbambas.org.

Xstrata. Ver página web:www.xstrata.career.com.au. 